\title{
Visual cues fail to attenuate deficits on a spatial-integration task following septal or fornical damage
}

\author{
THOM HERRMANN \\ University of Guelph, Guelph, Ontario, Canada \\ A. H. BLACK \\ McMaster University, Hamilton, Ontario, Canada \\ D. DOHERTY \\ University of Guelph, Guelph, Ontario, Canada \\ and \\ P. ELLEN \\ Georgia State University, Atlanta, Georgia 30303
}

\begin{abstract}
An attempt was made to reduce the deficit produced by a septal and/or fornical lesion on the 3 -table reasoning task through the addition of a visual cue. This procedure has previously been used to ameliorate deficits produced by these lesions in a variety of instrumental tasks. When the cue was used to indicate a particular location it had no effect on performance. When the cue was used to indicate the presence and location of food, the performance of fornically damaged subjects improved and the performance of control subjects dropped. However, the performances of both groups were comparable to levels achieved when the task was made into a discriminative learning situation. These results emphasize the qualitative distinction between spatial integration and spatial discrimination performance and gives support to the suggestion that the fornix is involved in spatial integration processes. On the other hand, the performance of septal-lesioned subjects was unaffected by the addition of the cue, suggesting a more severe deficit than loss of spatial integrative ability.
\end{abstract}

In previous studies (Herrmann, Black, Anchel, \& Ellen, 1978; Stahl \& Ellen, 1974), it was shown that septal and/or fornically damaged animals were unable to solve the integrative spatial problem on Maier's 3-table task. It will be recalled that, on this task, the animal is first given a preliminary exploratory experience over the three tables, is then fed on one of the tables, and finally, is given a test trial in which it must return to the table on which it had just been fed. Maier (1929) has suggested that a solution to the problem depends on the rat's ability to store and integrate each day's separate experiences. In fact, each day the feeding table and start table are randomly varied so that it is impossible for the animal to learn a particular route to the food.

Commonalities in the behavioral deficits following septal and fornical lesions have been reported in other tasks as well (cf. Isaacson, 1974). However, the literature is replete with references to the fact that the behavioral deficits on instrumental tasks and operant schedules following septal, fornix, or hippocampal lesions can be ameliorated by providing the animals an exteroceptive stimulus (cue light) which would indicate to the animals when to respond (Lubar \& Numan, 1973; O’Keefe \& Nadel, 1978; Srebro, 1974). The question then arises as to whether such a procedure would ameliorate the deficit on the 3-table task following either septal or fornical lesions. In other words, would placing an exteroceptive cue on the correct table during the feeding and testing phase of the problem facilitate the performance of brain damaged rats on this problem?

\section{METHOD}

\section{Subjects}

One hundred and twenty-eight male Long-Evans rats, obtained from the Guelph Psychology Department's breeding colony and housed individually in a light (light 0800-2000, dark 2000-0800) and temperature $\left(18 \pm 2^{\circ} \mathrm{C}\right)$ controlled limited-access colony, provide the data base of this study. Prior to the beginning of training, the subjects were reduced to $85 \%$ of ad-lib weight and maintained at that level for the duration of the experiment.

\section{Apparatus.}

The basic apparatus consisted of three pathways (each $1.15 \mathrm{~m}$ in length) that formed equal angles from a center point $(\mathrm{Y})$. The table at the end of each pathway differed in size and shape, but was.faced with wooden sides which prevented observation of one table from 
another. Entrance to each table was provided by an $8 \times 8 \mathrm{~cm}$ hole. The entire apparatus was painted flat black, elevated $.71 \mathrm{~m}$ above the floor, and housed in a $3.4 \times 3.4 \mathrm{~m}$ room. The room was lit by a 40-W incandescent light source located in one corner of the room. An Everready Lifelight model portable $(1.5 \mathrm{~V})$ light source was used as an additional cue in several conditions. The light source, when used, was suspended $1.5 \mathrm{~m}$ above either the correct or incorrect table and was visible from any other table or portion of the apparatus.

\section{Surgical Procedures.}

Prior to behavioral training, all animals were assigned randomly to one of three groups: septal lesions, fornical transected, or surgical control. The animals were anesthesized $(.36 \mathrm{mg} / \mathrm{kg}$ ) with sodium pentobarbital (Nembutal). Septal lesions were created by lowering the tip of a relatively blunt (therefore reducing vascular damage due to tearing or cutting) Kopf RFG 4 electrode to coordinate $\mathrm{AP}=7.5, \mathrm{~L} \pm 0, \mathrm{D} / \mathrm{V}+6.5$ from stereotaxic zero and heating the tip to $80^{\circ} \mathrm{C}$ for $15 \mathrm{sec}$. The fornix transections were produced using a specially designed knife which consisted of two juxtaposed stainless steel L-shaped blades. The horizontal section of each blade was $15 \mathrm{~mm}$ long and so arranged that the blades could be separated to a distance of $1 \mathrm{~mm}$. When the blades were closed, only the distal $12 \mathrm{~mm}$ of each blade made contact. The knife was permanently mounted on a tower, allowing the horizontal blades a lateral approach to the fornix. A small hole was made in the side of the skull at the following coordinates: $1 \mathrm{~mm}$ posterior to bregma and $3.9 \mathrm{~mm}$ below the surface of the skull. The open horizontal blades of the knife were then inserted through the lateral opening into the brain for a distance of $11 \mathrm{~mm}$ from the lateral surface of the brain. The blades of the knife were then closed for $30 \mathrm{sec}$ before being reopened; then the knife was removed. Control procedures consisted of anesthetizing the animals, placing them in the stereotaxic device, and opening their skulls in the manner of either the septal or fornical preparations.

Following behavioral testing, all animals were anethetized with a lethal dose of Nembutal and perfused with saline. The brains were removed, frozen, sectioned at 20 microns, conterstained with either (a) hemotoxlyn and eosin or (b) luxol methyl blue and cresyl violet, and examined.

\section{Behavioral Procedures:}

Prior to the initiation of testing, each subject was handled $10 \mathrm{~min}$ per day. This taming was followed by 5 days of training the animals to run down a straight pathway (similar in length and width to those in the experimental apparatus) for food. The basic format of the 3-table task is to give an exploratory experience, a feeding experience, and a test trial. On the test trial, the animal is expected to go to the table where it was just fed. The exploratory experience consists of a 15-min period during which the animals are allowed to run the entire unbaited apparatus. This initial experience was followed by 3 min during which the animals were placed on one of the tables and allowed to eat. During the feeding experience, the pathway system was removed so that no additional exploration was possible. Following feeding, the rat was removed from the apparatus. The apparatus was then thoroughly cleaned and the food and pathways were replaced. The animals are then tested by placing them on one of the remaining two tables and recording their choices. If an animal did not run to the table on which it was just fed, it was allowed to correct. One trial was given per day with a different combination of start and feeding tables used each day over a period of 24 days.

Modifications to this basic procedure consisted of placing the cue light over either the food table or the incorrect table either during the exploration, feeding experience, and test trial or during the feeding and test trial alone. Figure 1 illustrates the various experimental arrangements that were given each group of animals.

\section{RESULTS AND DISCUSSION}

\section{A sufficient number of animals were run for each}

cell of Figure 1 to contain eight animals. Animals were not used if their damage did not reach the criteria described below. Septal animals sustained bilateral damage to the medial, lateral, and dorsal septal nuclei, with less than $20 \%$ damage to adjacent structures (caudate and corpus callosum) and less than $10 \%$ damage to fornix, hippocampal commissure, and preoptic nuclear regions. Animals with fornical transections sustained damage to the septal area and rostral to the hippocampus. To be acceptable for this group, animals had to sustain less than $10 \%$ damage to the anterior thalamic nuclei, the stria medullaris, and the corpus callosom. In all instances, the extent of brain damage (Figure 2) was evaluated independently by the first three authors prior to examination of the behavioral findings. Little evidence of vascular damage

\begin{tabular}{|c|c|c|c|}
\hline CONDITION & EXPERIE & NCE & TEST \\
\hline Traditional & ${ }^{1} Y$ & 2 & $\mathbf{Y}^{\mathrm{G}}$ \\
\hline Goal Lit & $\mathbf{Y}^{\nabla}$ & $\underline{G}$ & $\mathbf{Y}^{c}$ \\
\hline Error Lit & ${ }^{\nabla} \mathbf{Y}$ & $\mathrm{E} \quad \mathrm{G}$ & \\
\hline Food Lit w-explore & $\mathbf{Y}$ & $\underline{G}^{\nabla}$ & \\
\hline Food Lit wo-explore & & $G^{\nabla}$ & \\
\hline
\end{tabular}

Figure 1. Diagramatic presentation of procedures used in each condition $(\mathbf{G}=$ goal; $\underline{G}=$ baited goal; $\mathbf{E}=$ potential error location; $\mathrm{S}=$ start location, $\nabla \equiv$ light cue)

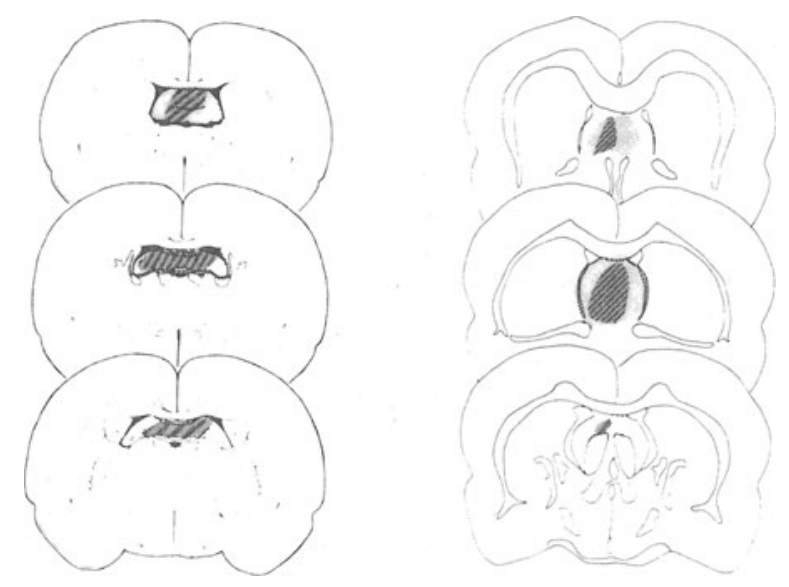

Figure 2. Representation of range of histologic results. Drawings present range (maximum $=$ lightly shaded; minimum $=$ darkly shaded) of extent of damage to septal-lesioned (right panel) and fornix-transected animals (left panel). 
following the midline septal penetration was noted in any of the rats by the examiners.

The major data of this experiement are shown in Table 1, which corresponds to the various groups depicted earlier in Figure 1. The data were initially analyzed using a repeated measures (day) multiple analysis of variance (Winer, 1971), whose significant differences were further differentiated through the use of the $t$ test ( $t$-crit) for differences among several means (Bruning \& Kintz, 1968). All statistically significant findings are indicated in the table.

It is apparent that adding a cue light to the 3-table problem had no significant effect on the reasoning performance when such a cue light was used during all conditions (exploration, feeding, and test trial). The addition of the cue light did not facilitate the formation of an integrative solution in either normals or braindamaged aninals. To this extent, it would seem that the 3-table problem taps into processes that are qualitatively different from processes in instrumental learning tasks where, indeed, the addition of an exteroceptive cue markedly improves performance of both normal and brain-damaged animals. It is of interest to point out that placing the exteroceptive cue light on the incorrect table did not impair performance. This fact suggests the relative independence of the integrative solution on specific extra-maze stimuli and again emphasizes the qualitative distinction between the processes implicated in the 3-table problem vis à vis those implicated in instrumental learning tasks such as mazes (Maier, 1929).

Of particular interest in this connection are the findings when the cue light was used only during the feeding and test trial. It will be noted from Table 1 that, under these circumstances, the performance of the unoperated animals was severely impaired when the feeding experience was preceded by an exploratory experience. When there was no prior exploration, then the normal animals had no difficulty in learning to go to the table containing the light. This would suggest that when the animals had a prior exploratory experi-

Table 1

Mean Correct Trials on First Choice

\begin{tabular}{llll}
\hline \multirow{2}{*}{ Condition } & \multicolumn{3}{c}{ Group } \\
\cline { 2 - 4 } \cline { 2 - 4 } Traditional & Control & Fornical & Septal \\
Goal Lit & 87.5 & $47.5^{* *}$ & $48.3^{* *}$ \\
Error Lit & 81.6 & $52.5^{* *}$ & $51.6^{* *}$ \\
Food Lit-Exploration Unlit & 79.6 & $58.3^{* *}$ & $47.6^{* *}$ \\
Food Lit-No Exploration & $60.8^{*}$ & $68.1^{*}$ & $55.8^{\dagger}$ \\
& 75.8 & $64.8^{*}$ & $50.8^{* *}$ \\
\hline
\end{tabular}

*Significantly different (t-crit $p<.05)$ when compared with that group's traditional condition. **Significantly different (t-crit $p<.05)$ when compared with control subjects in that condition. TSignificantly different $(t$-crit $p<.05)$ when septal control and fornical groups are compared on same condition. ence, the kind of learning that occurred during that experience [spatial or cognitive map (O'Keefe \& Nadel, 1978)] was not compatible with the learning acquired during the feeding experience ( $\mathrm{S}^{\mathrm{d}}$ learning), and, as a consequence, these two kinds of learning could not be integrated into a solution (Black \& Darymple, in press). However, when the task is simply reduced to a traditional discriminative learning task, by omitting the exploratory experience and just associating the food table with the light, then normal animals can learn to go to the lit table without too much difficulty, considering the fact that they are receiving only one trial per day.

This analysis allows us to understand the performance of the fornix-damaged animals. Table 1 shows that when the cue light was used only during the feeding and test trial, fornix rats were not influenced by whether or not they received a prior exploratory experience. In each instance, fornix rats went to the lit table $68.1 \%$ and $64.8 \%$ of the time, respectively; their performance did not differ from that of the controls. These findings would suggest that either removing the exploratory experience or producing fornix damage is sufficient to convert a spatial-integrative cognitive problem such as the 3-table task into a simple lightdiscrimination task. To this extent, additional support is given to the distinction between the processes involved in performance on a cognitive task as compared to those in an instrumental learning task. Furthermore, these findings lend major support to the O'Keefe-Nadel (1978) concept of the role of fornix in the organization of cognitive maps, since the presence of the exploration experience had no effect on the fornix rats, implying that these animals were, indeed, insensitive to the spatial-integrative aspects of the problem. The performance of the fornix-damaged animals stands in marked contrast to that of the normals when viewed in this light.

Finally, the refractoriness of the septal animals to any of the manipulations employed in this study was somewhat surprising. These observations would imply a more severe deficit in the case of the septal animals than simply a loss of spatial integrative ability as in the case of fornical animals. While the fornical animals could not solve on the basis of a spatialintegrative process, they could learn to go to the lit table. The septals, in contrast, could not learn to go to the lit table even when it contained food, or to avoid it when it did not contain the food. The specific basis for this enhanced deficit awaits further investigation.

\section{REFERENCES}

Black, A. H., \& Dalrymple. A. J. Instrumental multistage problem solving in the rat. Canadian Journal of Psychology, in press.

Bruning, J. L., \& Kintz, B. L. Computational handbook of statistics. New York: Scott Foresman, 1968. 
Herrmann, T., Black, A. H., Anchel, H., \& Ellen, P. A comparison of septal and fornical lesioned rats on the Maier three table reasoning task. Physiology \& Behavior, 1978, 20, 297-302.

IsaAcson, R. L. The limbic system. New York: Plenum, 1974.

Lubar, J. F., \& Numan, R. Behavioral and physiological studies of septal function and related medial cortical structures. Behavioral Biology, 1973, 8, 1-25.

MAIER, N. R. F. Reasoning in the white rat. Comparative Psychology Monographs, 1929, 6, 1-93.

O'KEEFE, J., \& NADEL, L. The hippocampus as a cognitive map. London: Oxford University Press, 1978.
SREBro, B. Visual cues in successive position reversals: Retention deficits following septal lesions. Physiology \& Behavior, 1974, 12, 639-646.

Stahl, J. M., \& Ellen, P. Septal lesions and reasoning performance in the rat. Journal of Comparative and Physiological Psychology, 1974, 87, 598-604.

Winer, B. J. Statistical principles in experimental design 12 nd ed.). New York: McGraw-Hill, 1971.

(Received for publication May 8, 1979; revision accepted November $19,1979$. 